\title{
Missense mutations of $B R C A 1$ gene affect the binding with p53 both in vitro and in vivo
}

\author{
BARBARA QUARESIMA*, MARIA C. FANIELLO*, FRANCESCO BAUDI, TELMA CRUGLIANO, \\ MADDALENA DI SANZO, GIOVANNI CUDA, FRANCESCO COSTANZO and SALVATORE VENUTA
}

Dipartimento di Medicina Sperimentale e Clinica, Università degli Studi di Catanzaro 'Magna Græcia', Viale Europa, Campus Universitario Germaneto, I-88100 Catanzaro, Italy

Received March 13, 2006; Accepted May 29, 2006

\begin{abstract}
Women with BRCA1 gene mutations have an increased risk for breast and ovarian cancer (BOC). Classification of missense variants as neutral or disease causing is still a challenge and has major implications for genetic counseling. $B R C A l$ is organized in an $\mathrm{N}$-terminal ring-finger domain and two BRCT (breast cancer C-terminus) domains, involved in protein-protein interaction. The integrity of the C-terminal, BRCT repeat region is also critical for BRCA1 tumor suppressor function. Several molecular partners of BRCA1 have so far been identified; among them, the tumor suppressor protein $\mathrm{p} 53$ seems to play a major role. This study was aimed at evaluating the impact of two missense mutations, namely the W1837R and the S1841N, previously identified in BOC patients and located in the BRCT domain of the BRCAl gene, on the binding capacity of this protein to p53. Co-immunoprecipitation assays of $E$. coli-expressed wild-type and mutated BRCTs challenged with a HeLa cell extract revealed, for the S1841N variant a significant reduction in the binding activity to $\mathrm{p} 53$, while the W1837R mutant showed an inverse effect. Furthermore, a clonogenic soft agar growth assay performed on HeLa cells stably transfected with either wild-type or mutant $B R C A 1$ showed a marked decrease of the growth in wild-type BRCA1-overexpressing cells and in BRCA1 $1^{\text {S1841N }}$-transfected cells, while no significant changes were detected in the

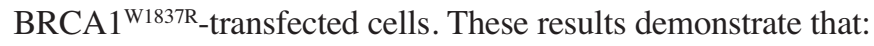
i) distinct single nucleotide changes in the BRCT domain of BRCA1 affect binding of this protein to the tumor suppressor p53, and ii) the two missense mutations here described are likely to play a role in breast tumorigenesis. We suggest that in vitro/in vivo experiments testing the effects of unclassified
\end{abstract}

Correspondence to: Professor Francesco Costanzo, Dipartimento di Medicina Sperimentale e Clinica, Università degli Studi di Catanzaro 'Magna Græcia', Viale Europa, Campus Universitario Germaneto, I-88100 Catanzaro, Italy

E-mail: fsc@unicz.it

${ }^{*}$ Contributed equally

Key words: BRCA1, BRCT, p53, missense mutations
BRCA1 gene variants should therefore be taken in to consideration and that increased surveillance should be adopted in individuals bearing these two BRCA1 missense alterations.

\section{Introduction}

$B R C A 1$ is a tumor suppressor gene whose germ-line mutations predispose to breast and ovarian cancer $(1,2)$. BRCAl encodes a nuclear phosphoprotein of about $220 \mathrm{Kd}$, functionally organized in domains located at the $\mathrm{N}$ - and C-terminus of the molecule (3). In particular, the $\mathrm{COOH}$-terminal BRCT domain is an evolutionarily conserved region characterized by hydrophobic clusters of amino acids that are thought to stabilize the three-dimensional structure of the protein (4). Along with the role in the stability of protein conformation, the BRCT domain is involved in protein-protein interactions with many different molecules (5). Several BRCA1 partners have been so far identified, including components of the DNA repair machinery (6), transcription factors (7-9), the transcriptional co-activator p300 (10) and p53 (11-13). Binding of BRCA1 to p53 requires a two-domain interaction, located at the C-terminus (aa 17561855), and at the N-terminus (aa 224-500) (11), respectively.

p53 is a nuclear protein of about $53 \mathrm{kDa}$ in size $(14,15)$, subdivided into four domains with distinct but interdependent functions $(16,17)$. Several triggers can activate p53 that, in turn, modulates a set of genes with protective activities for the cell, including those involved in control of the cell cycle and induction of apoptosis (17). The promoters of $p 21$ and Bax genes are some of these p53-activated targets. The physical association of p53 with BRCA1 increases p53-dependent transcription driven by these two promoters $(18,19)$. Mutations in the coding region of $\mathrm{p} 53$ have been identified in approximately $30-50 \%$ of breast cancer (20). Mutated p53 can no longer regulate BRCA1 expression in mammary tissue. Loss of either p53 or BRCA1 function leads to disruption of cell cycle check-points, accumulation of DNA damage, and ultimately to sporadic breast cancer (21).

It has been estimated that $B R C A 1$ gene mutations account for about $50 \%$ of hereditary breast cancer $(\mathrm{BC})$ and for about $80 \%$ of hereditary breast and ovarian cancer (BOC) (22). Interestingly, the majority of known cancer-causing BRCA1 mutations are localized in the $\mathrm{N}$ - and in the $\mathrm{C}$-terminal interaction domains $(2,23-26)$. While the pathogenic role of nonsense and frame shift mutations is well recognized in breast 
carcinogenesis, the impact of missense mutations is still to be defined.

The aim of our study was to evaluate the role played by two distinct missense mutations in the BRCT domain of the $B R C A 1$ gene on the binding of this protein to the partner $\mathrm{p} 53$ and to shed more light on their pathogenic role in breast carcinogenesis. Both mutations have been detected in $\mathrm{BC}$ and/or BOC cancer patients.

Wild-type and mutated BRCTs have been expressed in $E$. coli, challenged with a HeLa cell extract used as a source of p53 and then analyzed by Western blotting with an antip53 antibody. The results of the co-immunoprecipitation assay show a significant reduction in the binding to p53 by the BRCT domain carrying the $\mathrm{S} 1841 \mathrm{~N}$ amino acid change (of the order of $72 \%$ ), while the $\mathrm{BRCT}^{\mathrm{W} 1837 \mathrm{R}}$ mutation potentiates binding of BRCA1 to p53.

To further investigate the biological effects of BRCA1 missense mutations on the cellular phenotype, a clonogenic soft agar growth assay has been performed on HeLa cells stably transfected with wild-type and mutant BRCAl genes. Interestingly, wild-type BRCA1-overexpressing cells show a marked decrease of the clonogenic growth, while the BRCA $1{ }^{\text {W1837R }}$ transfected cells have a markedly different behavior, mimicking the untransfected HeLa cells. On the other hand, a significant reduction of cell growth is found in BRCA1 $1^{\text {S1841N }}$ transfected cells.

Our results provide new insights into the molecular mechanisms underlying the pathogenic role of single nucleotide mutations on BRCA1-dependent $\mathrm{BC}$ tumorigenesis and may be considered a valuable approach in the identification of genetic alterations potentially critical in the predisposition of individuals to cancer.

\section{Materials and methods}

Cell culture and transfection. HeLa cells were cultured as monolayer in Dulbecco's modified Eagle's medium (DMEM) (Life Technologies) supplemented with $10 \%(\mathrm{v} / \mathrm{v})$ fetal calf serum (Sigma), 100 units/l penicillin (Hyclone). Cells were grown at $37^{\circ} \mathrm{C}$ in a $5 \% \mathrm{CO}_{2}$ atmosphere. Parental HeLa cells were transfected with pcDNA 3.1 (empty vector transfected), pcDNA BRCA1wt, pcDNA BRCA1 W1837R and pcDNA BRCA1 S1841N using the calcium phosphate precipitation method. Cells were then selected in G418 $400 \mu \mathrm{g} / \mathrm{ml}$ (Invitrogen, Life Technologies).

Plasmids. The pcDNA3.1 plasmid containing the fulllength $B R C A 1$ gene (pcDNA BRCA1wt) was a gift of Dr M. Montagna. Site-directed mutagenesis of the BRCAl cDNA insert was obtained using the QuickChange Kit (Stratagene). The BRCAl mutants were generated with the following primers: forward 5'-GACCCGAGAGCGGGTGTTGGACA GTG-3' and reverse 5'-CACTGTCCAACACCCGCTCTCG GGTC-3' for pcDNA BRCA1 ${ }^{\text {w1837R; }}$; forward 5'-GTGTTGGA CAATGTAGCACTCTACC-3' and reverse 5'-GGTAGAGT GCTACATTGTCCAACAC-3' for pcDNA BRCA1 $1^{\text {s1841N }}$.

The wild-type and mutant $B R C A 1$ s encoding the region from aa 1537 to aa 1861 were generated with the following primers: forward 5'-GCCCGGGATGCAACAGCTGGAAG AG-3' and reverse 5'-AAGCTTTTAGCTGGGGATCTGGG-3' and cloned into the SmaI site of pQE-30 (Qiaexpress Kit, Promega). All constructs were verified by DNA sequencing. The DH5 $\alpha$ strain was used in cloning procedures.

p53-BRCA1 interaction in vitro. E. coli strains M15 (pRep4) harboring $\mathrm{pQE}$ BRCA $1^{\mathrm{wt}}$, $\mathrm{pQE}$ BRCA $1^{\mathrm{w} 1837 \mathrm{R}}$ and $\mathrm{pQE}$ BRCA $1^{\text {S1841N }}$ were grown to early logarithmic phase. After incubation with $1 \mathrm{mM}$ isopropyl- $\beta$-D-thiogalactopyranoside (IPTG) for $2 \mathrm{~h}$ at $37^{\circ} \mathrm{C}$, bacteria were lysed by sonication in buffer $\mathrm{H}$ (20 mM Hepes; $300 \mathrm{mM} \mathrm{KCl} ; 0.1 \mathrm{mM}$ EDTA; 0.1\% NP40; $1 \mathrm{mM}$ PMSF), and protein products were purified from the soluble extract fraction by centrifugation. The supernatants were incubated with Ni-NTA agarose for $2 \mathrm{~h}$ at $4^{\circ} \mathrm{C}$. Histidinetagged proteins were collected by centrifugation, washed four times with buffer $\mathrm{H}$ and incubated for $2 \mathrm{~h}$ at $4^{\circ} \mathrm{C}$ with $1 \mathrm{mg}$ of HeLa cell total extract (27). The beads were collected by centrifugation and washed four times with buffer $\mathrm{H}$. The proteins were electrophoresed on SDS/10\%-PAGE gels and transferred to a nitrocellulose filter (Immobilon ${ }^{\mathrm{TM}} \mathrm{P}$; Millipore). After addition of the blocking mixture [5\% (w/v) BSA in PBS, pH 7.4], the membrane was incubated with a 1:200 dilution of rabbit anti-human BRCA1 antibody (Santa Cruz) or of mouse anti-human p53 antibody (Santa Cruz) for $2 \mathrm{~h}$ at room temperature. Bound antibody was detected with antirabbit horseradish peroxidase-conjugated secondary antibody (1:5000) or anti-mouse horseradish peroxidase-conjugated secondary antibody (1:5000), respectively. Following three washes in PBS-Tween for 10 min each time, the membrane was developed by ECL-Western blot detection reagents according to the manufacturer's instructions (Santa Cruz).

Soft agar growth assay. Soft agar assay was performed using 5x10 ${ }^{4} \mathrm{HeLa}$ parental cells, HeLa/pcDNA 3.1, HeLa/ ${ }^{\mathrm{wt}}$ BRCA1, $\mathrm{HeLa} /{ }^{\mathrm{S} 1841 \mathrm{~N}} \mathrm{BRCA} 1$ and $\mathrm{HeLa} /{ }^{\mathrm{W} 1837 \mathrm{R}} \mathrm{BRCA} 1$ respectively, assayed for BRCA1 protein expression. They were suspended in $1.5 \mathrm{ml}$ of $0.5 \%$ Noble Agar (Difco, Kansas City, MO, USA), $100 \mathrm{mM}$ triptose phosphate buffer (Difco), 10\% FCS DMEM medium and layered on $7 \mathrm{ml}$ of similar buffer in 60-mm culture dishes. Plates were incubated at a constant temperature of $37^{\circ} \mathrm{C}$ with a humidified atmosphere of $5 \% \mathrm{CO}_{2}$ for 3 weeks and counted.

\section{Results}

Effects of BRCT missense mutations on the interaction with p53. The BRCT domain is a main target for cancer associated mutations; out of 891 (28) DNA variants so far reported in the BRCAl gene more than $22 \%$ are localized within this region. The BRCT domain is also one of the two domains responsible for the binding of BRCA1 with p53 (11). In order to assess the potential impact of BRCT missense mutations to the binding with the partner protein $\mathrm{p} 53$, two distinct variants (W1837R and S1841N) were introduced in the wild-type BRCT domain of BRCA1 cDNA by site-directed mutagenesis (Fig. 1). The wild-type and the mutagenized cDNA fragments (from nt 4611 to nt 5583), cloned in the His-tag pQE30 plasmid, were then expressed in E. coli, immobilized on Ni-NTA Agarose and used as bait against a total protein extract from HeLa cells transfected with a p53 expression vector. After extensive washing, p53 protein interacting with 


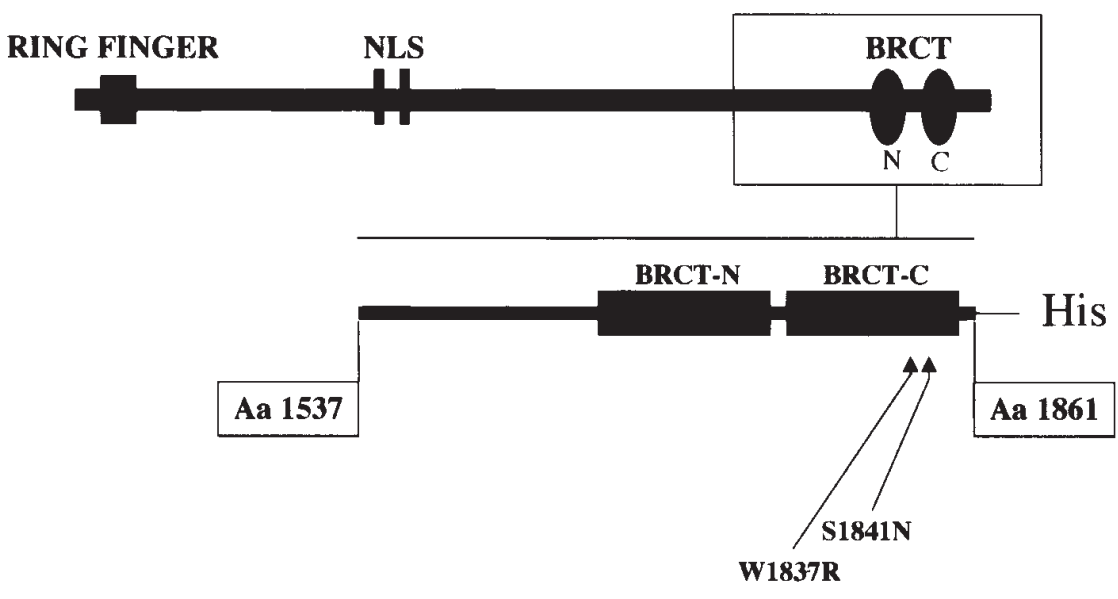

Figure 1. Domain structure of BRCA1. (Top), Schematic representation of full-lenght BRCA1 protein featuring the RING domain in the N-terminal region and BRCT domains in the C-terminal region. The region analyzed (aa 1537-1863) is contained in the box, which is enlarged and presented (bottom), BRCA1 C-terminal region (aa 1537-1863) His tagged. Mutations analyzed are depicted.

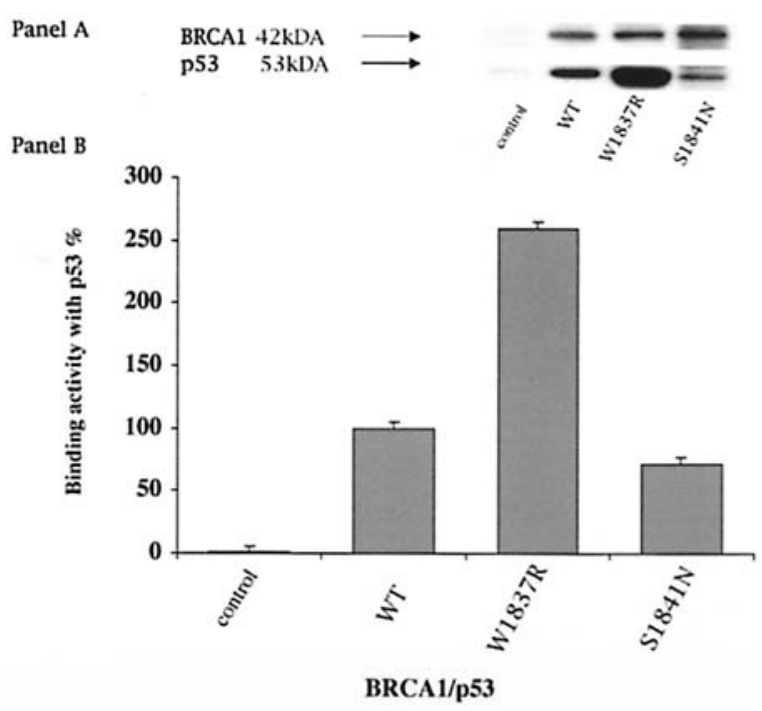

Figure 2. Interaction of p53 with histidine-tagged BRCA1. Panel A, Amounts of histidine-tagged wild-type (wt) or mutated BRCA1 proteins ('W1837R' and 'S1841N') were immobilized on Ni-NTA-agarose (upper panel), and challenged with total protein extract derived from HeLa cells as described in Materials and methods. After extensive washing, the amount of p53 protein interacting with wild-type or mutated BRCA1 proteins was determined by Western blot using a mouse anti-human p53 antibody (lower panel). The expected molecular masses of BRCA1 and p53 proteins are indicated on the left. Panel B, Quantitative analysis of the interaction between $\mathrm{p} 53$ protein and histidine-tagged wild-type or mutated BRCA1 proteins. Values are means for at least five independent experiments shown in (A) and they represent percentages $( \pm$ SEM) of densitometry values.

BRCA1 was assayed by Western blotting using a rabbit antip53 antibody, as shown in Fig. 2. Because the expression efficiency of mutant BRCA1 polypeptides in bacterial cells was not homogeneous, protein concentration in the immunoprecipitation assay was adjusted to contain equivalent amounts of BRCA1, as estimated by Western blot performed with a mouse anti-His-tag antibody (Fig. 2, panel A). The empty pQE30 vector (Fig. 2, panel A) and the untreated Ni-NTA agarose (data not shown), were used as negative controls. The relative intensity of the signal was quantified by densitometry scanning of the filter (Fig. 2, panel B). The results indicate that the two missense mutations in the BRCT domain do not abolish the binding of BRCA1 to p53, but are able to consistently alter the heterodimer complex formation. More specifically, in the S1841N allele, the reduced intensity of the chemiluminescence signal, compared to that of the wild-type allele, strongly suggests a defect in binding to p53, while the W1837R replacement consistently increases the interaction with p53 (on average about 2.5-fold), and/or the stability of the protein complex.

Effects of BRCA1 variants on colony formation in soft agar. The biochemical analysis of BRCTs mutant alleles indicates that nucleotide substitutions in BRTC domain modulate BRCA1/p53 interactions. Since both p53 and BRCA1 are related with control of cell growth and neoplastic transformation (29), we decided to test the growth properties of the mutant alleles by measuring their ability to form colonies in a soft agar assay.

To this end, we stably transfected HeLa cells with either the wild-type BRCAl cDNA (HeLa/wt BRCA1) or with the W1837R (HeLa/W1837R BRCA1) and S1841N (HeLa/S1841N BRCA1) mutated forms (Materials and methods). HeLa cells transfected with vector alone were used as control. The clones were selected with G-418 and the expression efficiency of wild-type and mutant BRCA1 His-tagged proteins was checked by Western blot analysis (data not shown). HeLa cells were subsequently layered on soft agar (Materials and methods) and the colonies were stained and counted after 3 weeks. Striking differences were observed in two independent selection experiments, both in number (Fig. 3) and size (data not shown) of colonies formed. The BRCA1 wild-type expressing clone produced a reduced number of colonies (about 30\%) compared to the ones generated by parental HeLa; approximately the same number of colonies was obtained with the S1841N allele (about $28 \%$ of the control). The size of the S1841N colonies was consistently smaller than the size of colonies expressing wt BRCA1 and parental control HeLa. On the other hand, the W1837R-expressing clone produced colonies comparable in size and number to those produced by parental HeLa. These experiments indicate that the expression of wt BRCA1 reduces the ability of HeLa cells to grow on soft 


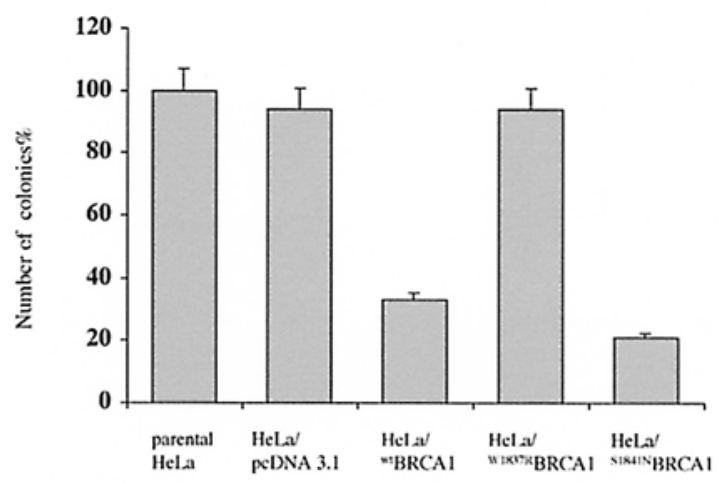

Figure 3. Soft agar growth assay. Quantitative analysis of in vivo clonogenic growth assay on HeLa cells stably transfected with either the wild-type BRCA1 cDNA (HeLa/wt BRCA1) or two distinct mutagenized BRCA1 cDNA (HeLa/ ${ }^{\text {W1837R }}$ BRCA1 and HeLa/S1841N BRCA1) (Materials and methods). Values are means for at least two independent experiments.

agar, as it does for expression of the S1841N allele. Conversely, the expression of the W1837R allele fully restores the ability of HeLa cells to grow without adhering to a solid matrix, to an extent comparable to that of parental cells.

\section{Discussion}

This study analyzed the potential role of $B R C A 1$ missense mutations identified in families with hereditary BC and BOC. While the pathogenic role of non-sense and frame shift germ line mutations is well recognized in $\mathrm{BC} / \mathrm{BOC}$ carcinogenesis, the impact of missense mutations, accounting for about $37 \%$ (28) of the overall BRCAl gene alterations, is still to be defined. This latter type of mutations, resulting solely in the substitution of a single aa residue, do not cause premature termination of the polypeptide but might alter critical physico-chemical properties potentially responsible for the stabilization the BRCA1 protein structure and/or its interactions with other proteins, thereby impairing the normal function and predisposing to BC.

Several approaches have been used to characterize missense variants at the BRCT domains. Williams et al (30) used a protease-based assay to directly assess the sensitivity of the folding of the BRCT domain to a set of truncations as well as single amino acid substitutions derived from BC screening programs; Gaiser et al (31) performed dynamics measurements on the NMR structure of the isolated C-terminal BRCT domain of human BRCA1; Mirkovic et al (32) made a structure-based assessment of missense mutations in human BRCA1, with the specific aim to increase the ability to identify cancerassociated variants, particularly those that abolish transcriptional activation.

In this report, we have analyzed the role of two missense mutations in the BRCT domain with respect to two parameters, i.e. the ability to bind p53 and the capacity in forming colonies in a soft agar assay. The results of protein-protein interaction analysis indicate that the $B R C A 1$ allele carrying the $\mathrm{S} 1841 \mathrm{~N}$ mutation significantly reduces binding to $\mathrm{p} 53$ when compared with the wt allele, while the W1837R modification produces an inverse effect. Thus, mutations affecting strictly contiguous amino acids may lead to opposite biological effects.
The crystal structure of rat BRCA1 tandem-BRCT region (PDB 1L0B) has been resolved (http://www.rcsb.org/pdb) (33). In this model, the BRCT domain can be subdivided in three distinct fragments [fragment 1, from ARG 1591 to GLN 1702 (112 aa); fragment 2, from LEU 1705 to GLU 1756 (52 aa); and fragment 3, from ARG 1776 to GLN 1801 (26 aa) and in three loop areas (L1a from 1688 to 1693; L1b from 1638 to 1641; L3 from 1788 to 1801)]. The loops 1a and $1 \mathrm{~b}$ are known to be involved in the interaction with p53 (33). The rat and human amino acid sequences show a high degree (about 70\%) of homology (30). In the sequence alignment, the human W1837 corresponds to the rat W1782 and the S1841 corresponds to the rat $\mathrm{S} 1786$. The $\mathrm{W} 1782$ residue, that is localized exactly in the middle of an $\alpha$ helix, shows a consistent degree of interaction with ASP1794 side chain, as well as with other amino acids, such as VAL1778, PHE1706 and VAL1783, located within a hydrophobic cleft. We suggest that the substitution of a non-polar aromatic side-chain with the basic amino acid ARG, directly in contact with the terminal loop L3, may dramatically modify the network of H-bonds in this segment of the BRCA1 molecule. As a consequence, important conformational changes may take place in the terminal residues of the L3 loop, and produce an increase in its conformational rigidity that may strengthen the binding with p53 and/or the stability of the protein complex. Concerning the SER1786, the $\mathrm{OH}$ group of its side chain interacts with TRP1782 and with ARG1793 with H-bonds and the amidic $\mathrm{H}$ interacts with VAL1783 residue, stabilizing the conformation of fragment 3. The SER to ASN transition introduces a strong steric effect, which may expose the loop L3 in a place potentially unfavorable to interact with p53. Moreover, the L1a and L1b loop areas may be affected, therefore contributing to the loss of affinity of the mutant BRCT to p53.

To shed more light on the molecular mechanisms underlying BC/BOC tumorigenesis triggered by the two BRCA1 mutations, only one of whom (W1837R) has been reported to be as a deleterious/high-risk variant (34), we have performed a clonogenic soft agar growth assay, a technique commonly used to test in vivo tumorigenicity (35).

The clonogenic soft agar growth assay performed on HeLa cells stably transfected with either wild-type or mutant BRCAI $\left(B R C A 1^{\mathrm{W} 1837 \mathrm{R}}\right.$ and BRCA1 ${ }^{\mathrm{S} 1841 \mathrm{~N}}$ ) reveals, in the wt-BRCA1overexpressing cells, a significant decrease of the clonogenic growth, according with the data of Thompson et al (36), while the BRCA $1^{\text {W1837R }}$-transfected cells mimic parental HeLa cells. A significant reduction of cell growth in soft agar is found in BRCA1 $1^{\mathrm{S} 1841 \mathrm{~N}}$-transfected cells. We believe that the conformational rigidity of mutant BRCA1 induced by the W-R substitution titrates out $\mathrm{p} 53$, which is in turn unable to exert its anti-oncogenic role; on the contrary, the S-N aa change might lose the interaction between BRCA1 and p53, making it highly unstable and therefore functionally impaired.

BRCA1 is known to bind to p53 with a bi-partite mechanism, involving both $\mathrm{N}$ - and C-terminus. Even though in this study only the C-terminal region of BRCA1 was challenged with p53, nevertheless we believe that the perturbations produced on the folding of the BRCT domain by the two distinct missense mutations are able, per se, to functionally impair the interaction between the two partners and the biological pathways regulated by this interaction. 
In conclusion, this study provides evidence that the BRCA $1^{\mathrm{W} 1837 \mathrm{R}}$ and BRCA1 ${ }^{\mathrm{S} 1841 \mathrm{~N}}$ variants play a pathogenic role in breast carcinogenesis and strengthen the concept that in vitro and in vivo assays devoted to test the effects of 'unclassified' BRCA1 mutants are essential for genetic counselling.

\section{Acknowledgements}

We thank Dr M. Montagna for providing us with the entire ORF of BRCA1. This study was supported by grants from MIUR (Ministero Università e Ricerca Scientifica e Tecnologica) COFIN 2003 and CLUSTER C-04, from Ministero della Salute, Ricerca Finalizzata 2004, and from AIRC (Associazione Italiana Ricerca sul Cancro).

\section{References}

1. Miki Y, Swensen J, Shattuck-Eidens D, et al: A strong candidate for the breast and ovarian cancer susceptibility gene BRCA1. Science 266: 66-71, 1994.

2. Friedman LS, Ostermeyer EA, Szabo CI, Dowd P, Lynch ED, Rowell SE and King MC: Confirmation of BRCA1 by analysis of germline mutations linked to breast and ovarian cancer in ten families. Nat Genet 8: 399-404, 1994

3. Chen Y, Farmer AA, Chen CF, Jones DC, Chen PL and Lee WH: BRCA1 is a $220-\mathrm{kDa}$ nuclear phosphoprotein that is expressed and phosphorylated in a cell cycle-dependent manner. Cancer Res 56: 3168-3172, 1996.

4. Bork P, Hofmann K, Bucher P, Neuwald AF, Altschul SF and Koonin EV: A superfamily of conserved domains in DNA damage-responsive cell cycle check-point proteins. FASEB J 11: 68-76, 1997.

5. Wang Y, Cortez D, Yazdi P, Neff N, Elledge SJ and Qin J: BASC, a super complex of BRCA1-associated proteins involved in the recognition and repair of aberrant DNA structures. Genes Dev 14: 927-939, 2000.

6. Wang Q, Zhang H, Guerrette S, Chen J, Mazurek A, Wilson T, Slupianek A, Skorski T, Fishel, R and Greene MI: Adenosine nucleotide modulates the physical interaction between hMSH2 and BRCA1. Oncogene 20: 4640-4649, 2001.

7. Anderson SF, Schlegel BP, Nakajima T, Wolpin ES and Parvin JD: BRCA1 protein is linked to the RNA polymerase II holoenzyme complex via RNA helicase. Nat Genet 19: 254-256, 1998.

8. Wang Q, Zhang H, Kajino K and Greene MI: BRCA1 binds c-Myc and inhibits its transcriptional and transforming activity in cells. Oncogene 17: 1939-1948, 1998.

9. Schlegel BP, Green VJ, Ladias JA and Parvin JD: BRCA1 interaction with RNA polymerase II reveals a role for hRPB2 and hRPB10alpha in activated transcription. Proc Natl Acad Sci USA 97: 3148-3153, 2000.

10. Pao GM, Janknecht R, Ruffner H, Hunter T and Verma IM: $\mathrm{CBP} / \mathrm{p} 300$ interact with and function as transcriptional coactivators of BRCA1. Proc Natl Acad Sci USA 97: 1020-1025, 2000.

11. Zhang H, Somasundaram K, Peng Y, Tian H, Zhang H, Bi D, Weber B and El-Deiry WS: BRCA1 physically associates with p53 and stimulates its transcriptional activity. Oncogene 16: 1713-1721, 1998.

12. Ouchi T, Monteiro AN, August A, Aaronson SA and Hanafusa H: BRCA1 regulates p53-dependent gene expression. Proc Natl Acad Sci USA 95: 2302-2306, 1998.

13. Chai YL, Cui J, Shao N, Shyam E, Reddy P and Rao VN: The second BRCT domain of BRCA1 proteins interacts with p53 and stimulates transcription from the $\mathrm{p} 21 \mathrm{WAF} 1 / \mathrm{CIP} 1$ promoter. Oncogene 18: 263-268, 1999.

14. Lane DP and Crawford LV: T antigen is bound to a host protein in SV40-transformed cells. Nature 278: 261-263, 1979.

15. Linzer DI and Levine AJ: Characterization of a 54K dalton cellular SV40 tumor antigen present in SV40-transformed cells and uninfected embryonal carcinoma cells. Cell 17: 43-52, 1979.

16. Stürzbecher HW, Brain R, Addison C, Rudge K, Remm M, Grimaldi M, Keenan E and Jenkins JR: A C-terminal alphahelix plus basic region motif is the major structural determinant of p53 tetramerization. Oncogene 7: 1513-1523, 1992.
17. Ko LJ and Prives C: p53: puzzle and paradigm. Genes Dev 10: 1054-1072, 1996.

18. Somasundaram K, Zhang H, Zeng YX, Houvras Y, Peng Y, Zhang H, Wu GS, Licht JD, Weber BL and El-Deiry WS: Arrest of the cell cycle by the tumour-suppressor BRCA1 requires the CDK-inhibitor p21WAF1/CiP1. Nature 389: 187-190, 1997.

19. Miyashita T and Reed JC: Tumor suppressor p53 is a direct transcriptional activator of the human bax gene. Cell 80: 293-299, 1995.

20. Greenblatt MS, Chappuis PO, Bond JP, Hamel N and Foulkes WD: TP53 mutations in breast cancer associated with BRCA1 or BRCA2 germ-line mutations: distinctive spectrum and structural distribution. Cancer Res 61: 4092-4097, 2001.

21. Katsama A, Sourvinos G, Zachos G and Spandidos DA: Allelic loss at the BRCA1, BRCA2 and TP53 loci in human sporadic breast carcinoma. Cancer Lett 150: 165-170, 2000.

22. Easton DF, Bishop DT, Ford D and Crockford GP: Genetic linkage analysis in familial breast and ovarian cancer: results from 214 families. The Breast Cancer Linkage Consortium. Am J Hum Genet 52: 678-701, 1993.

23. Futreal PA, Liu Q, Shattuck-Eidens D, et al: BRCA1 mutations in primary breast and ovarian carcinomas. Science 266: 120-122, 1994.

24. Gayther SA, Warren W, Mazoyer S, et al: Germline mutations of the BRCA1 gene in breast and ovarian cancer families provide evidence for a genotype-phenotype correlation. Nat Genet 11: 428-433, 1995.

25. Brzovic PS, Rajagopal P, Hoyt DW, King MC and Klevit RE: Structure of a BRCA1-BARD1 heterodimeric RING-RING complex. Nat Struct Biol 8: 833-837, 2001.

26. Shen D and Vadgama JV: BRCA1 and BRCA2 gene mutation analysis: visit to the Breast Cancer Information Core (BIC). Oncol Res 11: 63-69, 1999.

27. Quaresima B, Alifano P, Tassone P, Avvedimento VE, Costanzo FS and Venuta S: Human mismatch-repair protein MutL homologue 1 (MLH1) interacts with Escherichia coli MutL and MutS in vivo and in vitro: a simple genetic system to assay MLH1 function. Biochem J 371: 183-189, 2003.

28. Abkevich V, Zharkikh A, Deffenbaugh M, Frank D, Chen Y, Shattuck D, Skolnick MH, Gutin A and Tavtigian SV: Analysis of missense variation in human BRCA1 in the context of interspecific sequence variation. J Med Genet 41: 492-507, 2004.

29. Rosen EM, Fan S, Pestell RG and Goldberg ID: BRCA1 gene in breast cancer. J Cell Physiol 196: 19-41, 2003.

30. Williams RS, Chasman DI, Hau DD, Hui B, Lau AY and Glover JN: Detection of protein folding defects caused by BRCA1-BRCT truncation and missense mutations. J Biol Chem 278: 53007-53016, 2003.

31. Gaiser OJ, Ball LJ, Schmieder P, Leitner D, Strauss H, Wahl M, Kuhne R, Oschkinat $\mathrm{H}$ and Heinemann U: Solution structure, backbone dynamics, and association behavior of the C-terminal BRCT domain from the breast cancer-associated protein BRCA1. Biochemistry 43: 15983-15995, 2004.

32. Mirkovic N, Marti-Renom MA, Weber BL, Sali A and Monteiro AN: Structure-based assessment of missense mutations in human BRCA1: implications for breast and ovarian cancer predisposition. Cancer Res 64: 3790-3797, 2004.

33. Joo WS, Jeffrey PD, Cantor SB, Finnin MS, Livingston DM and Pavletich NP: Structure of the 53BP1 BRCT region bound to p53 and its comparison to the Brcal BRCT structure. Genes Dev 16: 583-593, 2002.

34. Phelan CM, Apic V, Tice B, Favis R, Kwan E, Barany F, Manoukian S, Radice P, van der Luijt RB, van Nesselrooij BPM, Chenevix-Trench G, kConFab, Caldes T, de la Hoya M, Lindquist S, Tavtigian SV, Goldgar D, Borg $\AA$, Narod SA and Monteiro ANA: Classification of BRCA1 missense variants of unknown clinical significance. J Med Gen 42: 138-146, 2005.

35. Shin SI, Freedman VH, Risser R and Pollack R: Tumorigenicity of virus-transformed cells in nude mice is correlated specifically with anchorage independent growth in vitro. Proc Natl Acad Sci USA 72: 4435-4439, 1975.

36. Thompson ME, Jensen RA, Obermiller PS, Page DL and Holt JT: Decreased expression of BRCA1 accelerates growth and is often present during sporadic breast cancer progression. Nat Genet 9: 444-450, 1995. 